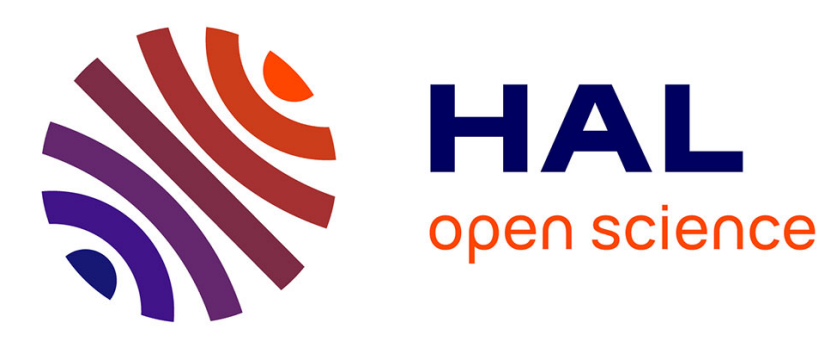

\title{
Are Dynamic Mechanistic Explanations Still Mechanistic?
}

Tarik Issad, Christophe Malaterre

\section{To cite this version:}

Tarik Issad, Christophe Malaterre. Are Dynamic Mechanistic Explanations Still Mechanistic?. PierreAlain Braillard; Christophe Malaterre. EXPLANATION IN BIOLOGY: AN ENQUIRY INTO THE DIVERSITY OF EXPLANATORY PATTERNS IN THE LIFE SCIENCES, 11, Springer, pp.Pages: 265-292, 2015, History Philosophy and Theory of the Life Sciences, 10.1007/978-94-017-9822-8_12 . hal-02196795

\section{HAL Id: hal-02196795 \\ https://hal.science/hal-02196795}

Submitted on 29 Jul 2019

HAL is a multi-disciplinary open access archive for the deposit and dissemination of scientific research documents, whether they are published or not. The documents may come from teaching and research institutions in France or abroad, or from public or private research centers.
L'archive ouverte pluridisciplinaire HAL, est destinée au dépôt et à la diffusion de documents scientifiques de niveau recherche, publiés ou non, émanant des établissements d'enseignement et de recherche français ou étrangers, des laboratoires publics ou privés. 


\title{
Are Dynamic Mechanistic Explanations Still Mechanistic?
}

\author{
Tarik Issad \\ INSERM U1016, CNRS UMR8104, Université Paris Descartes \\ tarik.issad@inserm.fr \\ Christophe Malaterre \\ Université du Québec à Montréal, CIRST \\ malaterre.christophe@uqam.ca
}

\begin{abstract}
A major type of explanation in biology consists of mechanistic explanations (e.g. Machamer et al. 2000, Kaplan and Craver 2011). The explanatory force of mechanisms is apparent in such typical cases as the functioning of an ion channel or the molecular activation of a receptor: it includes the specification of a model of mechanism and the rehearsing of a causal story that tells how the explanandum phenomenon is produced by the mechanism. It is however much less clear how mechanisms explain in the case of complex and non-linear biomolecular networks such as those that underlie the action of hormones and the regulation of genes. While dynamic mechanistic explanations have been proposed as an extension of mechanistic explanations (e.g. Bechtel and Abrahamsen 2010), we argue that the former depart from the latter in that they do not draw their explanatory force from a causal story but from the mathematical warrants they give that the explanandum phenomenon follows from a mathematical model. By analyzing the explanatory force of mechanistic explanation and of dynamic mechanistic explanation, we show that the two types of explanations can be construed as limit cases of a more general pattern of explanation - Causally Interpreted Model Explanations - that draws its explanatory force from a model, a causal interpretation that links the model to biological reality, and a mathematical derivation that links the model to the explanandum phenomenon.
\end{abstract}

Keywords Mechanisms - models - explanatory force - mechanistic explanation - dynamic mechanistic explanation - mathematical explanation.

T. Issad

Institut National de Santé et de Recherche Médicale, INSERM U1016, Institut Cochin, 22 rue Méchain, 75014 Paris, France.

C. Malaterre $(\square)$

Département de philosophie, UQÀM, Case postale 8888, Succursale Centre-Ville, Montréal (QC), H3C 3P8, Canada. 


\section{Introduction}

It is now often taken for granted that a major type of explanation in biology consists of mechanistic explanations (ME's). It is indeed often impossible to read a current scientific paper in biology without stumbling upon the concept of mechanism: mechanisms are found in articles dealing with subjects as diverse as regulation of food intake, transmission of nerve influx, cell division, cell growth, programmed cell death, production by mitochondria and chloroplasts of energy-rich molecules and so forth. It has been argued that the explanatory force of a mechanistic explanation - that is to say, what makes a mechanistic explanation explanatory - comes from displaying a mechanism while showing how this mechanism produces the phenomenon of interest. In this context, mechanisms are generally construed as particular types of models that include entities carrying out certain sets of activities and organized in such a way that they produce the phenomenon of interest (e.g. Machamer et al. 2000, Glennan 2002, Kaplan and Craver 2011). The explanatory force of ME's is claimed to be apparent in such canonical examples as the functioning of an ion channel involved in neuron firing or the molecular activation of a receptor. In this contribution, we question the explanatory force of ME when targeted at complex biological systems. More specifically, we argue that if the explanatory force of ME's is to be equated with displaying a mechanism and showing how this mechanism produces the phenomenon of interest, then such ME's are only possible with quite simple biological mechanisms. In the case of complex mechanisms - which are mechanisms that typically involve feedback loops and non-linear interactions (e.g. Bechtel and Abrahamsen 2010) - the explanatory force of proper explanations of phenomena produced by these mechanisms does not come from displaying the mechanisms and showing how these mechanisms produce the phenomena of interest, but rather from the use of dynamic mathematical models, thereby leading to specific dynamic mechanistic explanations (DME's). While it is a distinguishing feature of such mathematical models to be modally stronger than mechanisms or laws (Lange 2012), their use in complex biological systems highlights the very limited explanatory force of ME's. If there is anything distinctive to ME's, then their scope of application is actually quite restricted to fairly simple mechanisms, and their extension to complex biological systems (e.g. Bechtel and Abrahamsen 2010) is far from obvious when it comes to specifying the source of their explanatory force. We argue that, in the case of such complex systems, explanations are not mechanistic but follow a more general pattern of "Causally-Interpreted Model Explanations" (CIME, as we propose to call them). In the following section, we review what is usually defined as a mechanistic explanation (ME) and we highlight what makes mechanistic explanations explanatory: a model of mechanism and a causal story. We then show, in section 3, how these elements of explanatory force are effective in the case of simple biological mechanisms, and how they are ineffective in the case of complex biological mechanisms. We 
illustrate our argument by looking at the case of the action of insulin on glucose homeostasis. In section 4, we show that the explanatory force of explanations of phenomena produced by complex biological mechanisms (DME's) does not come from a causal-story but from their mathematical component and its stronger modality. As a consequence, we argue in section 5, that, while ME's are perfectly explanatory in the case of simple biological systems, DME's are no longer mechanistic since they do not draw their explanatory force from the same types of elements. In section 6, we show how the different elements of explanatory force of ME's and DME's can be reconstructed into three core elements that consist of a model, a causal interpretation and a mathematical derivation. We develop the notion of "Causally Interpreted Model Explanation" around those three elements and argue that ME's and DME's can be construed as limit cases of CIME's.

\section{Mechanistic explanation and its explanatory force}

An explanation presupposes that there is something to be explained - the explanandum - and something that does the explaining - the explanans. In the case of mechanistic explanation (ME), the explanandum is usually taken to be a phenomenon produced by a mechanism, while the explanans usually consists of displaying the mechanism and of showing how this mechanism produces the phenomenon in question. Let us be more specific.

A mechanism is usually defined as a set of parts and activities organized in such a way that they produce the phenomenon in question. For instance, Craver proposes to define mechanisms as "entities and activities organized such that they exhibit the explanandum phenomenon" (2007, p. 6). And for Glennan, "a mechanism for a behavior is a complex system that produces that behavior by the interaction of a number of parts, where the interactions between parts can be characterized by direct, invariant, change-relating generalizations" (2002, p. S344). Mechanisms so construed are real things: they are composed of real parts that engage in real activities or change-relating generalizations. The way we formalize our knowledge about a given mechanism is typically by defining a model of this mechanism: a model of mechanism. Indeed, Glennan defines the notion of "mechanical model" which is a description of a mechanism (2002, p. S347), and Craver proposes to distinguish a range of "models of mechanisms", ranging from "how possibly models" to "how actually models". Note that, in the scientific and philosophical literature, mechanisms are not always distinguished from their representational counterparts. Indeed, mechanistic explanations are often directly framed in terms of mechanisms. Nevertheless, the distinction matters if we wish to be precise about what a mechanistic explanation consists of. We will therefore use "model of mechanism" to mean a representation of a "mechanism".

Similarly, when one speaks about explaining a phenomenon, one 
presupposes a description of this phenomenon, this description typically being given under the form of a proposition (or a set of propositions). In the case of mechanistic explanation, the phenomenon that constitutes the explanandum is supposed to be produced by a mechanism.

A mechanistic explanation consists somehow in linking a description of the phenomenon (produced by a mechanism) to a representational model of this mechanism. Of course, much hinges on what is meant by "linking" a description of a phenomenon to a model of mechanism. It has been argued that in a mechanistic explanation,

"intelligibility arises [...] from an elucidative relation between the explanans (the set-up conditions and intermediate entities and activities) and the explanandum (the termination condition or the phenomenon to be explained) [...]. Descriptions of mechanisms render the end stage intelligible by showing how it is produced by bottom out entities and activities. To explain is not merely to redescribe one regularity as a series of several. Rather, explanation involves revealing the productive relation" (Machamer et al., 2000, p. 22).

In other words, providing a mechanistic explanation is a matter of showing how the entities of the mechanism produce the phenomenon through carrying out their respective activities. This "productive" relation is in turn often understood in a causal sense, with causation understood in a manipulationist sense (e.g. Craver, 2007). A mechanistic explanation therefore explains a phenomenon (produced by a mechanism) by showing how the phenomenon is causally brought about by the entities and activities of the mechanism.

In practice, this is done in two steps, first by exhibiting a model of the mechanism, second by showing how this model of mechanism causally accounts for the explanandum phenomenon. This model includes entities and activities that, ideally, refer to the real entities and activities of the real mechanism (and less ideally to plausible or even to possible ones - see (Craver, 2007, p. 139)), while specifying also their spatial and temporal organization, all of this being relevant to the phenomenon to be explained. Along these lines, Craver details five normative elements that a model of a mechanism must necessarily fulfill in order to contribute to a mechanistic explanation: (i) the model of mechanism must account for all aspects of the explanandum phenomenon, (ii) the model of mechanism must be based on components and activities that are real, (iii) these activities must be causal (in a manipulationist sense), (iv) the entities and activities are spatially and temporarily organized, (v) the entities and activities are all relevant with respect to the explanandum phenomenon (for more details, see (Craver, 2007, pp. 161-162)). These normative elements summarize what we expect of a model of a mechanism that does some explaining. We indeed expect that the entities and activities mentioned in the model of mechanism relate to some real entities and activities, and that they thereby somehow tell us something about the real world out there. We expect that these entities and activities are indeed relevant - and not superfluous - when it comes to explaining the phenomenon in question. We 
expect that the model of mechanism can indeed account fully for the explanandum. And that the entities and activities are organized and causally meaningful is also something that makes sense if explaining entails showing how things work. Yet even if it fulfills all these normative elements, a model of a mechanism alone falls short of being an explanation. Indeed, a list of entities and activities, even supplemented with their spatio-temporal organization, rarely is explanatory in itself. What needs to be shown is how the mechanism produces the phenomenon of interest.

Hence a second step in a mechanistic explanation that consists in showing how the entities and activities that are represented in the model causally interact so as to produce the very phenomenon to be explained ${ }^{1}$. In other words, one needs to show how the explanans (the model of the mechanism) is linked to the explanandum (the phenomenon produced by the mechanism) ${ }^{2}$. What appears to matter in this second step is to somehow animate the model of the mechanism so as to reveal the ways in which the represented entities interact with one another through their respective activities and in function of their organization so as to reproduce the phenomenon which is the target of the explanation. When we say "animate", we mean that a mechanistic explanation appears to involve some kind of rehearsing of the unfolding of the activities of the different entities that compose the mechanism. It is as if we were to run through each of the different causal links in the right sequential order. The explanatory force of a mechanistic explanation thereby stems from the intelligibility that arises when one rehearses this sequence of causal interactions and realizes that this sequence ends up in the phenomenon to be explained. For instance, an explanation of the movement of $\mathrm{Na}+$ ions through the neuronal membrane during the phenomenon of the action potential - a canonical example - may involve the following passage:

At $\mathrm{V}_{\text {rest }}$ [the membrane potential at rest], a positive extracellular potential holds the $\alpha$ helix [a subunit of a trans-membrane molecular complex known as the $\mathrm{Na}^{+}$channel] in place. Weakening that potential, which happens when the cell is depolarized, allows the helix to rotate out toward the extracellular side (carrying a "gating charge"

1 It could be argued that the causal account or "causal story" of how the entities and activities bring about the explanandum phenomenon is an integral part of the model of the mechanism, as proposed for instance by Glennan $(2005, \mathrm{p}$. 446). We argue here that distinguishing the causal story from the model reveals two distinct dimensions of the explanatory force of mechanistic explanation, as we specify at the end of this Section 2.

2 In the covering law model of explanation, the explanation takes the form of an argument whose premiss is the explanans and conclusion is the explanandum. The explanans is thereby linked to the explanandum by means of a deductive inference. It could be argued that mechanistic explanations are explanatory in just the same argumentative way, the description of the explanandum phenomenon being deduced from the description of the mechanism. Yet mechanistic philosophers specifically insist that mechanistic explanation is not an argument for "the explanation lies not in the logical relationship between these descriptions but in the causal relationships between the parts of the mechanism that produce the behavior described" (Glennan, 2002, p. S348). What matters therefore in a mechanistic explanation is to somehow show how the explanans causally produces the explanandum. 
as positively charged amino acids move outward). This rotation, which occurs in each of the $\mathrm{Na}^{+}$channel's subunits, destabilizes the balance of forces holding the channel in its closed state and bends the pore-lining S6 region in such a way as to open a channel through the membrane. Another consequence of these conformation changes is that the pore through the channel is lined with hairpin turn structures, the charge distribution along which accounts for the channel's selectivity to $\mathrm{Na}^{+}$(Craver, 2007, p. 119).

This excerpt clearly aims at showing how things work inside the mechanism and how this results in the production of the explanandum phenomenon. It mentions entities (" $\alpha$-helix", "hairpin turn structures" etc.) that engage in a range of activities ("holding in place", "rotating", "bending" etc.) according to a specific spatial and temporal organization (which appears in the way the account runs). And it is typically this type of causal account that is expected to supplement a model of mechanism in order to constitute a truly explanatory mechanistic explanation. It has even been proposed that the way such causal mechanistic accounts are explanatory is by relating the interactions of the mechanism's components to familiar experiences, somehow "by an extension of sensory experience with ways of working" (Machamer et al., 2000, p. 22).

The explanatory force of a mechanistic explanation therefore appears to come in two steps: (1) the exhibition of a model of mechanism, that refers to real and relevant entities and activities, and whose behaviour reproduces the explanandum phenomenon, and (2) a causal story, that provides intelligibility to the model of mechanism by describing how the entities and activities of that mechanism work together so as to produce the explanandum phenomenon. More formally, the explanatory force of mechanistic explanations can be made more specific in the following way:

A mechanistic explanation (ME) explains a phenomenon (P) produced by a system (S) in virtue of fulfilling the following two necessary and sufficient conditions:

$(M M)$ Displaying a model of mechanism that represents a real mechanism in $\mathrm{S}$, with its entities, activities and spatio-temporal organization,

(CS) Rehearsing a causal story that enumerates the cause-effect relationships taking place in the mechanism up to the production of the phenomenon $\mathrm{P}$.

These conditions explicate how mechanistic explanations gain their explanatory force. Such explanations are deemed explanatory in virtue of displaying a model of mechanism (that tells something about the world), and of rehearsing how this model produces the explanandum through a sequence of causal links. ${ }^{3}$ In the next section, we discuss the explanatory force of

3 The two conditions of explanatory force we specify here do not specifically commit to the details of what constitutes a model of mechanism. For reasons of convenience, we use a terminology inspired from (Machamer, Darden, \& Craver, 2000), yet the argument could be adapted to others such as (Glennan, 2005) for instance. Similarly, the two conditions are not linked to any specific account of causation, even if manipulationism as per (Woodward, 
mechanistic explanation and show that this explanatory force, while being indeed effective in the case of simple mechanisms, becomes ineffective in the case of complex mechanisms.

\section{Mechanistic explanation in the face of increasingly complex biological mechanisms}

There are many examples of mechanistic explanations that have already been discussed in the philosophical literature. The neuronal action potential, the chemical transmission at synapses, the synthesis of proteins are all canonical examples of mechanistic explanations. In what follows, we focus on another biochemical mechanism: the mechanism of insulin action. As will become apparent, this mechanism is interesting because it lends itself well to a mechanistic explanation, but does so only to a certain limit.

Insulin is a hormone, that is to say a molecule secreted in the blood circulation by an organ or a tissue, and capable of acting on another tissue. Discovered during the first half of the 20th century, insulin is produced by the pancreas during ingestion of a meal. It has long been known that one of the main function of insulin is to permit the utilization and/or storage of the nutrients ingested during meals (See Fig. 1). Insulin plays a crucial role in the regulation of glucose homeostasis, and more specifically in keeping concentration of glucose in the blood (glycaemia) within a narrow range, around a basal value of 0.8-1 gramme per liter. Dysfunctions in the regulation of glucose homeostasis can lead to chronic hyperglycaemia, which deleterious effects will eventually lead to the development of overt diabetes. One of the main effects of insulin is to stimulate glucose uptake by a number of insulin target cells, which then metabolize glucose or store it either as glycogen (a glucose polymer) in liver and muscle cells, or in the form of fat in adipose tissue. This permits to rapidly bring back glycaemia at its basal level, and to avoid the development of chronic hyperglycaemia. In this paper, we will examine more specifically the action of insulin on one of its main target tissues, the adipose tissue. One of the main functions of the adipose cells is to store nutrients in the form of lipids, that will be released in the blood circulation during periods of starvation.

2003) is often used in the mechanistic literature. 


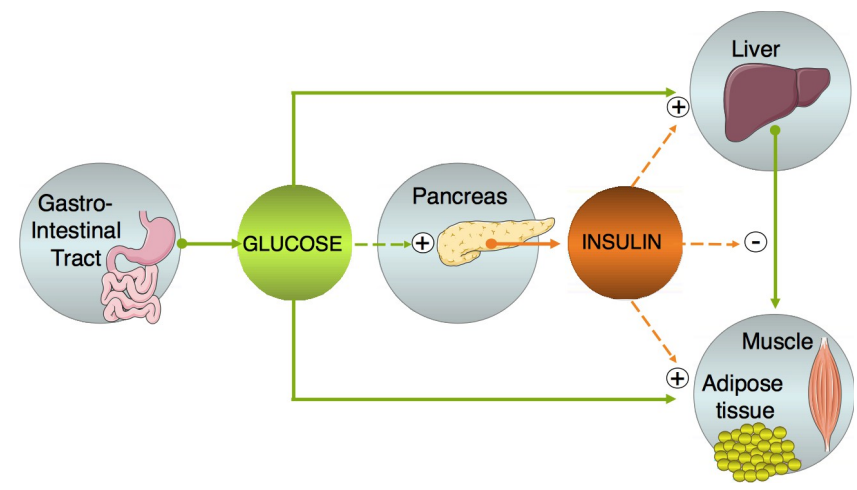

Figure 1. High level representation of the glucose-insulin control system.

At the beginning of the $1980 \mathrm{~s}$, a first step in the understanding of the mechanism of insulin action was crossed, when Cushman and Wardzala proposed that insulin stimulated glucose uptake by the adipocyte by inducing the relocalization of the "glucose transport system" from an intracellular compartment to the plasma membrane (Cushman \& Wardzala, 1980). This relocalization permitted to explain how insulin increased the activity of glucose transport at the surface of the cell, and thereby increased glucose uptake (see Fig. 2).

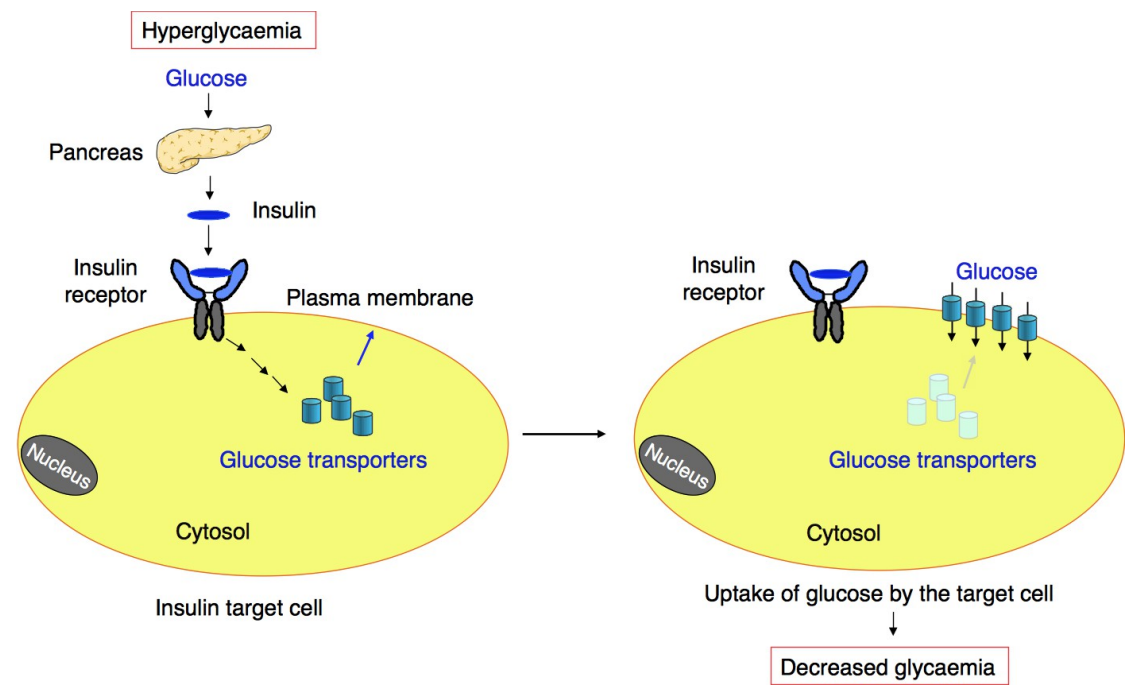

Figure 2. Insulin-induced relocalization of glucose transport systems

Further investigations revealed the entities bearing these activities (insulin receptor, glucose transporter) and the intermediary entities and activities that link insulin binding on its receptor to the movement of glucose transporters from an intracellular location towards the plasma membrane. Because numerous biological processes involve phosphorylation of proteins (a reversible modification consisting of the addition of phosphate groups on proteins), several investigators, using radioactive phosphate, studied the phosphorylation state of intracellular proteins following insulin stimulation (Belsham, 
Brownsey, Hughes, \& Denton, 1980; Smith, Rubin, \& Rosen, 1980). It was thus shown that a phosphorylating activity was associated with insulin action, resulting in increased incorporation of phosphate into a number of entities (intracellular proteins). It was then soon realized that the insulin receptor, in addition to its insulin binding activity located on the extra-cellular side of the cell surface, also possesses a phosphorylating activity, located inside the cell (M. Kasuga et al., 1982; Masato Kasuga, Zick, Blithe, Crettaz, \& Kahn, 1982). Surprisingly enough, the first target of this phosphorylating activity (tyrosine kinase activity) turned-out to be the insulin receptor itself, which autophosphorylates on tyrosine amino-acids upon insulin stimulation. Once phosphorylated on tyrosines, the insulin receptor is fully active and can phosphorylate intracellular proteins on tyrosines (See Fig. 3).

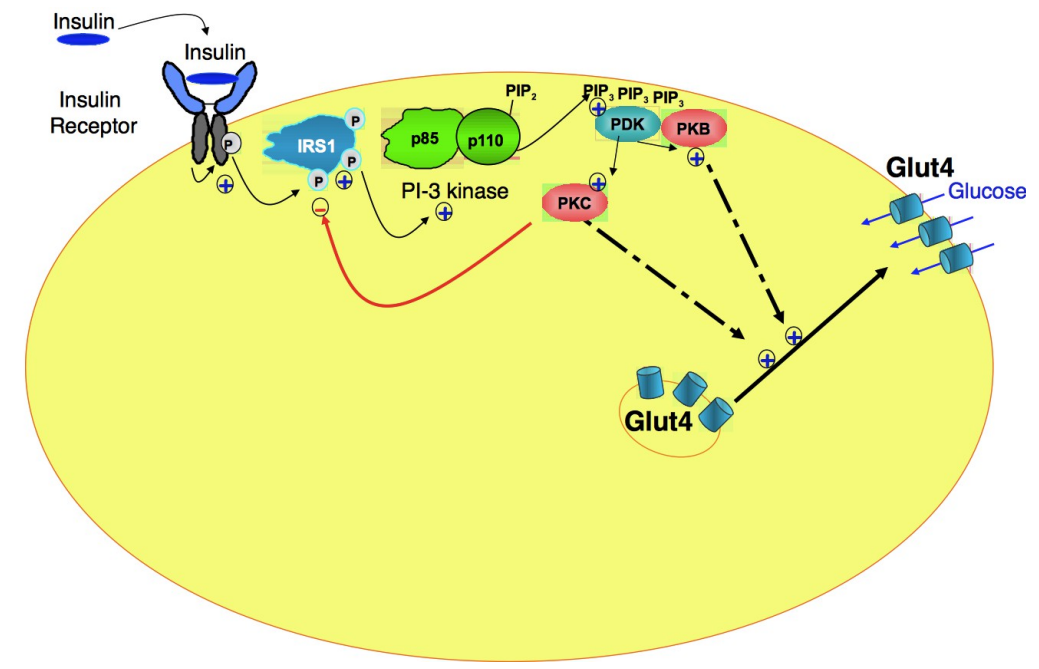

Figure 3. Mechanism of insulin action with regards to the activation of glucose transporters.

During the eighties and nineties, the generalization of the tools of molecular biology (determination of DNA and corresponding protein sequences) permitted the identification of a large number of entities involved in insulin action downstream of the receptor (Combettes-Souverain \& Issad, 1998). In addition, part of these proteins (e.g., the domain bearing the tyrosine kinase activity of the insulin receptor in its inactive (Hubbard, Wei, Ellis, \& Hendrickson, 1994) and active forms (Hubbard, 1997)) were crystallized, thereby bringing important insight into the tri-dimensional structure of these entities.

One of the proteins that bind phosphorylated IRS-1 is the Phosphatidyl Insositol-3 kinase constituting of a regulatory (p85) and a catalytic (p110) (See Fig. 3). The regulatory subunit binds to phosphorylated tyrosines of IRS-1, and this induces a stimulation of the catalytic activity of p110. We therefore have again a proper enzymatic activity and an interaction between IRS-1 and p85, that can as well be defined as a binding activity. The enzymatic activity of p110 
is a lipid-kinase activity, which phosphorylates lipids (Phosphatidyl Inositol-2 phosphate or PIP2) located on the inner face of the cell membrane. Once phosphorylated, these lipids (Phosphatidyl Inositol-3 Phosphate or PIP3) constitute anchorage platforms for membrane proteins with enzymatic activities (the serine/threonine kinases PDK, PKB and PKC $\zeta / \lambda$ ). Relocalisation of these protein kinases at the plasma membrane permit phosphorylation of AKT and PKC by PDK, resulting in stimulation of their activities (Combettes-Souverain \& Issad, 1998). Activated PKB and PKC then return into the cytosol and phosphorylate intracellular proteins, thereby releasing membrane vesicles containing glucose transporters (now identified at the molecular level as membrane channels denominated Glut4) from an intracellular location and their translocation to the plasma membrane (Hoffman \& Elmendorf, 2011). Insertion of Glut4 in the membrane then involves fusion of Glut4 containing vesicles with the plasma membrane, through complex and not completely defined "geometrico-mechanical" events, including movement and guidance of these vesicles through remodelling of cytoskeleton filaments, anchorage of these vesicles at the plasma membrane via the binding of proteins located on the surface of the vesicle to proteins located on the inner face of the plasma membrane, and finally fusion of the membranes allowing insertion of Glut4 in the plasma membrane.

The downstream action of insulin with regards to the insertion of glucose transport elements in the membrane of adipose cells is thereby explained mechanistically. The explanatory force of this explanation comes from the exhibition of a proper model of mechanism and from the causal story that accompanies it. The model of mechanism that is depicted above (as per Fig. 3) is proper in the sense that it satisfies the requirements that a model of mechanism that is present in a mechanistic explanation ought to satisfy: it refers to entities (IRS1, PI-3K, PDK, PKB, PKC, Glut4 etc.) that possess activities (catalyzing, binding, phosphorylating etc.) and that display both a spatial and temporal organization (some of these entities are located at the membrane of adipose cells, others within the cells, still others move from one place to another so as to enter into contacts with one another; the activities are carried out in a specific sequence of events). Simple negative feedback loops (e.g. phosphorylation of IRS1 by PKC) can also be included in the model without loosing its intelligibility. The entities and activities that the model refers to are taken to be real entities and activities, that are all causally relevant to accounting for the glucose intake of adipose cells upon stimulation by insulin. Furthermore, the model fully accounts for the phenomenon in question, including for its normal functioning under normal conditions as well as its dysfunctioning under a set of degraded or inhibitory conditions.

The explanatory force of this mechanistic explanation also comes from the causal story that accompanies the model of the corresponding mechanism (most of the above paragraphs of the present Section). It is this causal story that shows how the mechanism produces the explanandum phenomenon. The causal story thereby brings intelligibility to the model by revealing how the 
explanandum phenomenon causally follows from the entities and activities (and their spatio-temporal organization) that figure in the explanans model.

It is therefore fair to say that in this case, the mechanistic explanation works fine by virtue of fulfilling the two conditions of explanatory force that we have specified above for mechanistic explanations (ME): the condition of displaying a model of mechanism $(M M)$ and the condition of rehearsing a causal story $(C S)$. Like in many other examples of mechanistic explanation, the model of the mechanism and the causal story that accompanies it deliver the right intelligibility when it comes to explaining the explanandum phenomenon. However, a most interesting feature of this mechanism with respect to a discussion of the explanatory force of mechanistic explanation is that many of its entities and activities happen also to be linked to numerous other entities and activities located within the same adipose cells.

Indeed, careful investigations at each step of the mechanism resulted in the discovery of an important number of new entities: IRS-2,3,4,5 and 6, different isoformes of PI-3 kinase displaying different functions, a considerable number of regulatory proteins bearing enzymatic activities capable of dephosphorylating proteins as well as PIP3 (protein- or lipide-phosphatases), proteine kinases capable of phosphorylating and modulating the activities of different entities, as well as other proteins devoid of enzymatic activity but capable of binding to active sites of kinases and thereby blocking their catalytic activity (Carracedo \& Pandolfi, 2008; Lacasa, Boute, \& Issad, 2005; Nouaille et al., 2006; Virkamäki, Ueki, \& Kahn, 1999) (See Fig. 4). These entities are linked together through stimulatory or inhibitory interactions, resulting in a much more complex model of mechanism than previously.

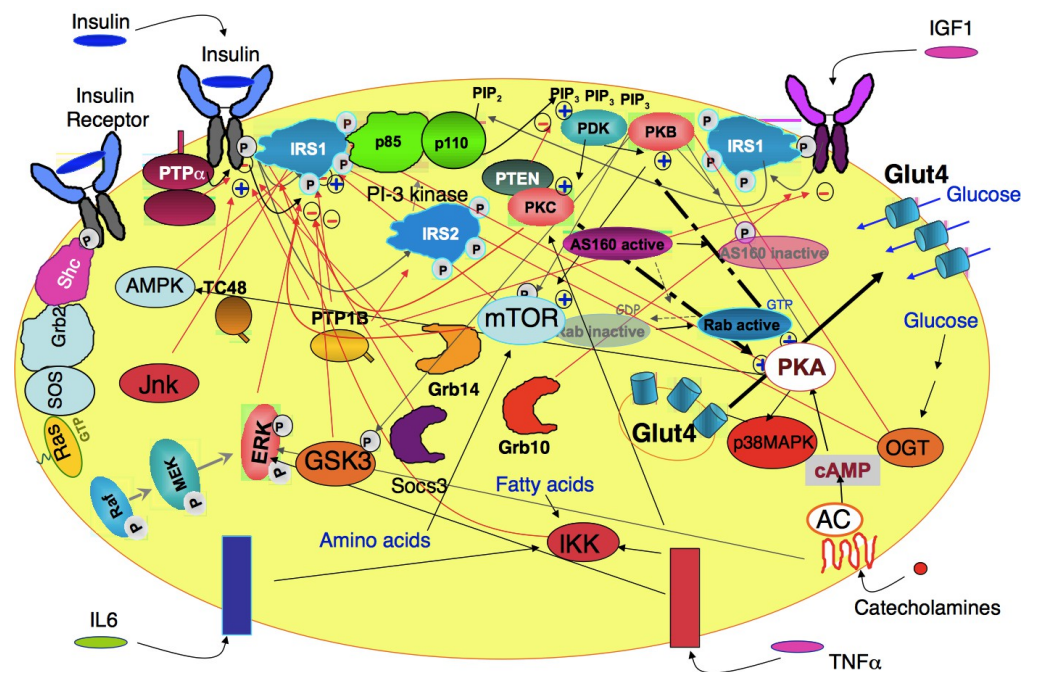

Figure 4. Mechanism of insulin action completed with some of the known interacting entities and activities within adipose cells (this model still represents a very light version of the information available to date). 
This complexity is further increased due to the discovery of new activities that are carried either by the original entities that are part of the simple mechanism of insulin action (as in Fig. 3) or by the new entities that complete this model (as in Fig. 4). Indeed, it is a known fact that a single entity can display different activities, because it can bear different domains with different functions (e.g., the insulin receptor, which has an insulin binding domain and a tyrosine kinase domain). But furthermore, the same domain within an entity can also display different activities, depending on the partner it interacts with. For instance, a lipid-kinase such as PI-3 Kinase (which phosphorylates PIP2 into PIP3) turnsout to be also a protein kinase (Hunter, 1995), whereas a lipid-phosphatase such as PTEN (which dephosphorylates PIP3 into PIP2) is also a protein phosphatase (Vazquez \& Sellers, 2000). More recently, it was shown that OGT, which glycosylates proteins according to glucose availability, is also a protease, capable of proteolytic cleavage of its substrates (Capotosti et al., 2011).

The complexity of the model of mechanism depicted in Fig. 4 is further increased by the fact that insulin triggers at the same time other mechanisms involving some of the entities described here as well as other entities not mentioned in this scheme, and resulting in other biological effects (protein synthesis, gene expression, etc...).

In addition, because adipose cells in living organisms are not isolated as they are in experimental set-ups, they are continuously impacted by a blood stream that contains not only insulin but also numerous hormones, which act upon the considered cell through numerous receptors, capable of triggering different mechanism that may interfere with the mechanism elicited by insulin. The concentrations of these hormones in the blood vary during the day (circadian rhythms) and according to the physiological state (nutritional state, physical activity, stress, etc.). These hormones affect the activities of some of the entities involved in the mechanism of insulin action. For instance, catecholamines (produced during a stress or under fasting conditions) bind to a family of hormone receptors (adrenergic receptors) that trigger different mechanisms capable of inhibiting several steps of insulin action in adipocytes (Tarik Issad, Combettes, \& Ferre, 1995), while activating other steps (Gerhardt, Gros, Strosberg, \& Issad, 1999; Moule et al., 1997). Other hormones, growth factors or cytokines, sometimes secreted by the adipose cell itself (autocrine factors), can also, at any time, activate or inhibit some of the entities involved in insulin action. In addition, several metabolites (fatty acids, glucose, aminoacids...) can also affect the activity of these entities. For instance, it is interesting to note that part of the glucose taken up by the cell will be used to directly modify some of the entities through O-glycoyslation (a reversible reaction analogous to phosphorylation that leads to addition of sugar on serine or threonine residues of des proteins). These glycosylations can modify several of the players involved in insulin action (IRS-1, PKB,...), thereby modulating their activities (sometimes by competing with the phosphorylation induced by insulin on the same serine or threonine residues) (T. Issad, Masson, \& Pagesy, 2010; Tarik Issad \& Kuo, 2008). 
Confronted with such a complexity, it seems legitimate to question the explanatory value of a mechanism, since it is becoming impossible to have a comprehensive view of the behaviour of the different parts working together. It is in such cases that the causal story $(C S)$ condition of explanatory force appears to break down: a causal rehearsal that would take into account such a complexity is no longer possible.

\section{When mechanistic explanations become dynamic}

To deal with such complex bio-systems, scientists may recur to different dynamic modelling techniques and simulation tools, including, for instance, ordinary differential equations (ODEs), process calculi, boolean networks, Bayesian networks, Petri nets, bipartite graphs, stochastic equations (see, e.g., Baldan, Cocco, Marin, \& Simeoni, 2010; de Jong, 2002; Deville, Gilbert, van Helden, \& Wodak, 2003; Mandel, Palfreyman, Lopez, \& Dubitzky, 2004; Wiekert, 2002 for some surveys). These different modelling techniques may be quantitative or qualitative, discrete or continuous, deterministic or probabilistic, and are frequently put to use in the context of imprecise and often incomplete knowledge. The choice of modelling techniques depends on the extent of the available data, but also on the nature of the molecular processes at stake (e.g. chemical reaction, complex formation, binding process, transcriptional regulation, etc), and on the chosen level of abstraction (for instance, a model of gene activation may include all different steps from transcription to translation, while another one may simply account for activation state). When much is known about the bio-system under scrutiny, and in particular when most kinetic data are available, scientists often turn to ODE-models as a means of gaining insight into the dynamic behaviour of the system. These models are then analyzed using bifurcation and phase plane analysis tools, and through numerical analyses run on computer simulation softwares (e.g., MATLAB, MATHEMATICA, SCILAB etc.).

Acknowledging the importance of such dynamic modelling tools in biology, Bechtel, Abrahamsen and Kaplan have proposed to extend the concept of mechanistic explanation so as to include what they call "dynamic mechanistic explanations" (Bechtel \& Abrahamsen, 2010; Kaplan \& Bechtel, 2011). A dynamic mechanistic explanation is an explanation that supplements a mechanism with a dynamic modelling. For instance, in the case of circadian rhythms (Bechtel \& Abrahamsen, 2010), it has been argued that a proper explanation of this phenomenon includes both a mechanism (whose components are specific molecular compounds interacting with each other in specific ways, such as promoting or inhibiting the synthesis of each other) and a dynamic modelling of this mechanism (that makes it possible to assess the time-related variations of key molecular compounds and therefore to account for the phenomenon at stake). So construed, dynamic mechanistic explanations 
involve both a mechanistic model ("mechanistic decomposition of the system into parts and operations"), and a mathematical model (typically from "computational modelling or dynamical systems theory") which are tightly coupled together in the sense that "some or all of the variables and terms in the mathematical model correspond to properties of identified parts and operations" (Bechtel \& Abrahamsen, 2012).

As Bechtel and colleagues have stressed, the display of the mechanism itself is not sufficient to provide an explanation of why there are circadian rhythms: one must appeal to dynamic modelling. Indeed, the time-related behaviour of the concentrations of the different molecules that figure in the mechanism can be accounted for by a set of three differential equations. Solving this set of equations numerically, that is to say typically by running a computer simulation, provides estimates of the time-related variations of each one of the different molecular concentrations. And, in this particular case, numerical simulations have shown that concentrations display oscillatory patterns with a period relatively close to 24 hours, hence an explanation of circadian rhythms $\mathrm{s}^{4}$. This has led Bechtel and colleagues to propose an extension of the mechanistic view of explanation: "Mentally rehearsing operations sequentially is not sufficient to determine how such a mechanism will behave, and the basic mechanistic account must be extended in the direction of dynamic mechanistic explanation in which computational modelling and dynamic systems analysis is invoked to understand the dynamic behaviour of biological mechanisms". (Bechtel, 2011, p. 554, our italics). A key question is how one construes such an extension.

One way to tackle this question is to look at how mechanistic explanations (ME's) on the one hand and dynamic mechanistic explanations (DME's) on the other gain their explanatory force. Recall that the explanatory force of a ME comes from the fulfillment of the two conditions $(M M)$ of displaying a model of mechanism, and $(C S)$ of rehearsing a causal story of the production of the explanandum phenomenon (see Section 2). On the other hand, if we follow Bechtel and colleagues, it seems that the explanatory force of a DME comes from (1) displaying a model of mechanism, and (2) providing a mathematical dynamic modelling of this mechanism that accounts for the explanandum phenomenon, and which is such that some or all of its variables and terms correspond to properties of identified parts and operations of the mechanism (Bechtel \& Abrahamsen, 2012). While the first element corresponds to the $(M M)$ condition above, the second explanatory element is of a different kind: it consists in specifying a mathematical model, in solving it - typically by means of numerical methods - and in showing how the solutions fit with the explanandum phenomenon. The explanatory force of DME's can therefore be made more specific in the following way:

4 For the sake of brevity, we over-simplify this example. See (Bechtel \& Abrahamsen, 2010, 2012) for more details. 
A dynamic mechanistic explanation (DME) explains a phenomenon (P) produced by a system (S) in virtue of fulfilling the following two necessary and sufficient conditions:

$(M M)$ Displaying a model of mechanism that represents a real mechanism in S, with its entities, activities and spatio-temporal organization,

(MA) Displaying a mathematical model such that some or all of its variables and terms correspond to properties of identified parts and operations of the mechanism, and providing mathematical warrants that its resolution/simulation fits $\mathrm{P}$.

The explanatory force of a DME is therefore not coming from the very same elements as the explanatory force of a ME. While a DME and a ME share the $(M M)$ condition of displaying a model of mechanism, the $\mathrm{ME}$ gains explanatory force by rehearsing a causal story (condition $C S$ ), while the DME gains explanatory force by providing and solving a mathematical modelling (condition $M A$ ).

Somewhat contrary to what Bechtel and colleague propose, it is difficult to see how DME's could constitute an extension of ME's since the explanatory force of ME's include an element that is not even present in the case of DME's, namely the condition $(C S)$ of rehearsing a causal story. How can one make sense of such an "extension"? It seems that we have here two possibilities. The first one would be to extend the concept of "mechanistic explanation" such that this concept should include DME's in addition to traditional ME's. If we follow this route, the explanatory force of a mechanistic explanation* would come either from the explanatory elements of a traditional $\mathrm{ME}$ or from the explanatory elements of a DME. ${ }^{5}$ Put more formally, we would have the following explication of the explanatory force of a mechanistic explanation*:

A mechanistic explanation* explains a phenomenon $(\mathrm{P})$ produced by a system $(\mathrm{S})$ in virtue of fulfilling the following condition: $(M M) \wedge[(C S) \vee(M A)] \quad{ }^{6}$

Such an option leads to defining a mechanistic explanation as a disjunction of two types of explanations that only share one common explanatory feature: the $(M M)$ condition. It would therefore seem that the key explanatory element of a mechanistic explanation comes from being based on the display of a mechanistic model. Yet this option, we argue, strongly weakens the traditional construal of a mechanistic explanation, since it removes an essential part of it that consists precisely in showing how the phenomenon is causally produced by the mechanism. As such, this would run contrary to the idea that a key feature of a mechanistic explanation is to exhibit causal relevance (e.g. Craver, 2007,

5 We have added a * to "mechanistic explanation" to distinguish this new extended concept from the traditional and narrower concept of mechanistic explanation that we have initialed (ME) everywhere else in the paper.

6 With the conditions $(M M),(C S)$ and $(M A)$ as previously defined. 
chap. 2).

The second possibility to make sense of an extension of mechanistic explanation in the direction of dynamic mechanistic explanation could be to criticize our account of what constitutes the explanatory force of a DME, and argue that the causal story condition $(C S)$ ought also to be included. In this case, ME's and DME's would share the two core explanatory elements $(M M)$ and $(C S)$. Note that, as a result, DME would not truly be an extension of ME, but rather a special type of ME whose explanatory force would come from the adjunction of a further condition, namely a mathematical modelling as described in $(M A)$. Yet, is this a viable option? Do DME's indeed lend themselves to causal story telling and do they gain explanatory force that way? We think not, and will argue the case with the help of an example that builds on the explanation of the insulin action we have started to describe above (Section 3). In a nutshell, we argue that, in the case of DME's, causal stories fall short of showing what is precisely expected of a causal story, namely how the system under study indeed causally produces the explanandum phenomenon.

\section{Explanatory force in the case of complex biological systems}

We have seen earlier that the translocation of glucose transporters from the cytoplasma to the cell membrane, thereby activating glucose uptake, is properly explained by a specific mechanistic explanation based on a model of mechanism and an associated causal story within which insulin plays a triggering causal role. Yet, the activation of glucose transporters is but one of the numerous mechanisms that happen to be at work in the broader phenomenon of glucose homeostasis. Recall that glucose homeostasis is the capacity of the human organism to keep its concentration of glucose in the blood (glycaemia) within a narrow range, around a basal value of 1 gramme per litre. Understanding how this is achieved is important as it may shed light on major dysfunctions such as chronic hyperglycaemia and diabetes. Explaining glucose homeostasis is however no trivial matter, as this phenomenon involves several organs, tissues and numerous molecular compounds and interactions across the whole body. Body-level models have been developed that link the concentration of glucose in the blood to the concentration of insulin, the latter inducing storage or release of glucose by the liver and its uptake by muscle and adipose tissue (e.g. Dalla Man, Rizza, \& Cobelli, 2006). Such body-level models have also been linked to tissue-level models, and in particular to models of the adipose tissue with regards to the action of insulin onto the activation of glucose transporters and glucose storage (e.g. Nyman et al., 2011). These tissue-level models are based on the mechanism of insulin action that we have seen earlier, but have also been detailed further so as to take into account nonlinear interactions between insulin molecules and insulin receptors at the 
surface of adipose cells (e.g. Brännmark, Palmér, Glad, Cedersund, \& Strålfors, 2010; Kiselyov, Versteyhe, Gauguin, \& De Meyts, 2009). This has led to the study of multi-level models as a means of explaining the action of insulin onto glucose homeostasis by unraveling a cascade of interactions, from the binding on insulin to its receptor, to the activation of glucose transporters, and finally the absorption of glucose by the adipose tissue and its effect on the whole glucose-insulin control system (Nyman et al., 2011). Such a model is depicted in Fig. 5.

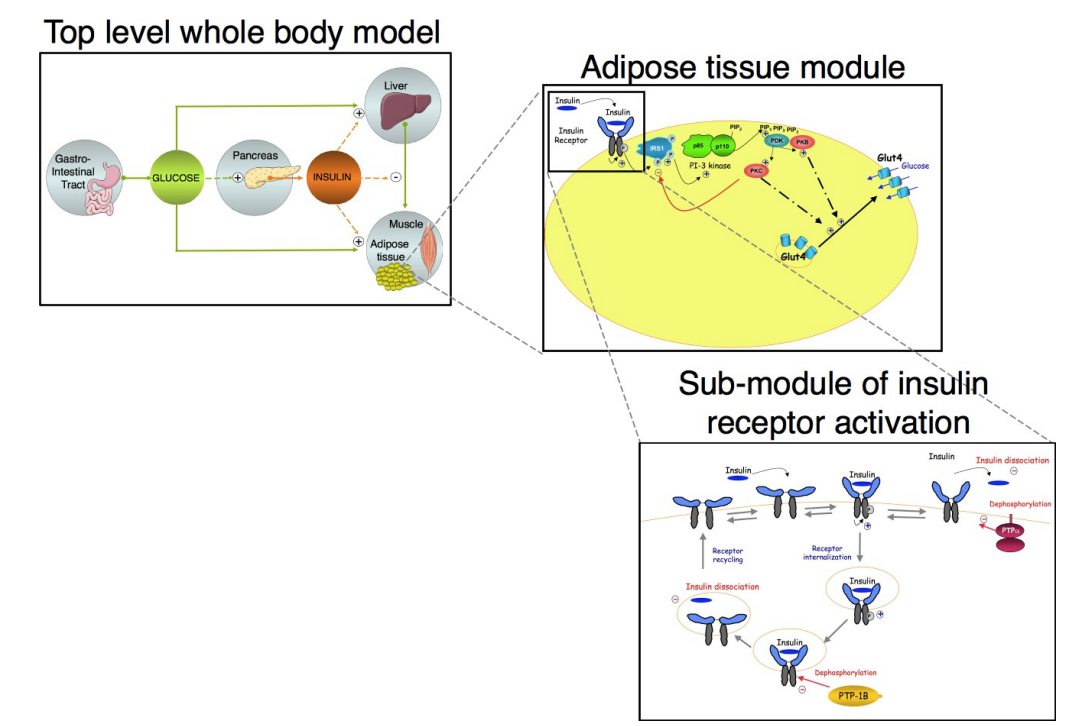

Figure 5. Multi-level dynamic modelling of the action of insulin on glucose homeostasis (adapted from Nyman et al., 2011).

It can be argued that what we have here is nothing but a classic "hierarchically organized mechanism", that is to say a mechanism involving several levels of finer-grained mechanisms (e.g. Craver, 2007, Chapter 5). And we grant that such a hierarchical multi-level model can be said to be a model of mechanism in the sense that it includes entities, activities and some form of spatio-temporal organization. An explanation that would be based on such model would therefore draw explanatory force from meeting the $(M M)$ condition. Recall however that the key question is whether such explanation would be explanatory by means of rehearsing a causal story, hence fulfilling also the $(C S)$ condition.

Before examining this question, let us analyze in more details the purported explanation that scientists put forward here (Nyman et al., 2011). The claim made by Cedersund, Strålfors and their colleagues is that such multi-level model explains the behaviour of the human organism with respect to glucose homeostasis as controlled by the adipose tissue. Following meal ingestion, the 
glucose concentration in the blood increases. ${ }^{7}$ This triggers the secretion of insulin by beta-cells in the pancreas and produces an "insulin signal." This insulin signal is then decoded by the liver and by the adipose tissue. It is this latter adipose tissue that is the focus of the model. The presence of insulin in the adipose tissue triggers a cascade of events, from the binding of insulin to insulin receptors, to the translocation of glucose transporters and the uptake of sugar by adipose cells. In turn, this uptake of sugar decreases the concentration of sugar in the blood, enabling the concentration of sugar to return to normal. The explanandum is why does the blood concentration return to normal after an increase due to meal ingestion? More precisely, what needs to be explained is the time-dependent concentration of glucose. The explanandum therefore is a dynamic feature of the human organism: the plotted curve of sugar concentration that has been measured experimentally as a function of time (See Fig. 6). And the scientists' claim is that their multi-level model explains precisely that: why glucose concentration has the time-dependent curve it has. ${ }^{8}$

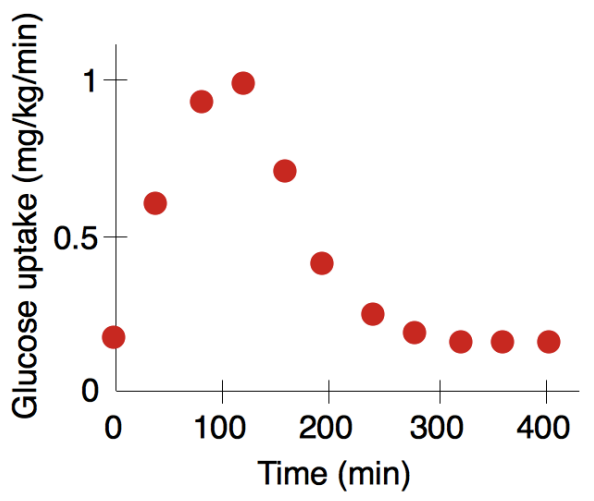

Figure 6. Plotted curve of experimentally measured sugar concentration as a function of time (adapted from Nyman et al., 2011).

The multi-level model tackles this question by exhibiting a hierarchy of models of mechanisms at the levels of the organism, the adipose tissue and the insulin receptor. All of these models are looked at from a time-dependent perspective: what matters is what happens as a function of time. And they are all non-linear, involving numerous feedback mechanisms, including some that run across levels. ${ }^{9}$ The way the different entities and activities of these models

7 Glucose homeostasis is not just involved during meal ingestion but also during fasting. For the sake of simplicity, we do not cover this aspect of the phenomenon, as this is not central to our philosophical argument.

8 To be accurate, the model aims at accounting not only for the time-variation of the concentration of glucose, but also for the time-variations of other compounds that can be measured experimentally, such as the \% of insulin receptors and of the \% of IRS1 that are phosphorylated in response to different insulin concentration variations.

9 For instance, Cedersund and Strålfors have included a "blood flow effect" linking the whole-body model and the adipose tissue model, so as to account for the fact that blood 
are represented is by means of a large set of ordinary differential equations (ODE's) that account for the variation over time of the concentrations of the different compounds as a function of their interactions with each other. For instance, the time-derivative of the concentration of insulin receptors is a function of the concentration of phosphorylated insulin receptors, of the concentration of insulin receptors and of the concentration of insulin (see Fig. 7 for a larger set of such ODE's).

$$
\begin{aligned}
& \dot{I R}=k 1 b \cdot I R p-k 1 f \cdot I R \cdot \text { insulin }-k 1 \text { basal } \cdot I R \\
& \dot{I R} p=-k 1 b \cdot I R p+k 1 f \cdot I R \cdot \text { insulin }+k 1 \text { basal } \cdot I R \\
& I \dot{R} S=k 2 b \cdot I R S p-k 2 f \cdot I R S \cdot I R p \\
& I \dot{R} S p=-k 2 b \cdot I R S p+k 2 f \cdot I R S \cdot I R p \\
& P \dot{K} B=k 3 b \cdot P K B p-k 3 f \cdot P K B \cdot I R S p \\
& P \dot{K} B p=-k 3 b \cdot P K B p+k 3 f \cdot P K B \cdot I R S p \\
& \text { GLUTT }=k 4 b \cdot G L U T 4 p m-k 4 f \cdot G L U T 4 \cdot P K B p \\
& \text { GLUTT } 4 p m=-k 4 b \cdot G L U T 4 p m+k 4 f \cdot G L U T 4 \cdot P K B p \\
& \text { glucose uptake }=k_{\text {glut } 1} \cdot \frac{\text { glucose }}{K m_{G 1}+\text { glucose }}+k_{\text {glut } 4} \cdot \frac{\text { glucose }}{K m_{G 4}+\text { glucose }} \cdot \text { GLUT4pm }
\end{aligned}
$$

Figure 7. Example of a set of ODE's used in one of the models of (Nyman et al., 2011).

Using ODE's is a mathematical way of encapsulating the causal knowledge that the variation of the concentration of certain entities is produced by the presence of other entities, at specific rates (reflected by kinetic constants). In a way, each ODE tells a causal story. Yet, these causal stories, even when patched together into a larger causal story do not lead to the production of the phenomenon to be explained. Recall that the explanandum phenomenon is the variation of sugar concentration as a function of time. At best, the causal story relates the variation of sugar concentration as a function of the concentration of other entities and of the variations of these concentrations or, more generally speaking, of other variables. The only way to untangle the many functional dependencies is to either analytically solve the set of ODE's when this is mathematically tractable, or to numerically estimate the solution functions by running computer simulations of the set of ODE's with the help of a computing softwares such as MATLAB, MATHEMATICA, SCILAB or others. The output of these computer simulations is a set of functions that represent the values of each variable as a function of time. Among these, one will have the simulated values of sugar concentration as a function of time. And if this curve matches with certain margins of error - the experimentally measured one, one will claim to have reproduced the explanandum phenomenon, and thereby to have explained it (see Figure 8). Yet, where is the causal story in this account?

flow may affect the availability of glucose and insulin in the local interstitial tissue surrounding the adipocytes, which in turn may effect glucose uptake by the adipocytes. For more details, see (Nyman et al., 2011). 
If causal stories are indeed possible when it comes to interpreting the individual ODE's that link the different variables with each other, such causal stories stop as soon as one steps into the process of mathematically solving the set of ODE's. Indeed solving the set of ODE's involve numerous technical steps which make it extremely difficult to see how any causal story could be rehearsed at all. For instance, in the very simple case of a set of first order linear differential equations, finding solutions typically involves writing the system of equations under a matrix form and finding the eigenvalues and eigenvectors of that very matrix. These different mathematical steps, many of which are very intricate and technical, relying in turn on sophisticated mathematical theorems, make it difficult to see how any causal story could make sense there. And of course, sets of differential equations can be much more tricky than that, requiring one to revert to "complex eigenvalues", to "repeated eigenvalues", to "undetermined coefficients", to techniques of "variation of parameters", to "Laplace Transforms", not to mention the many different tools involved in modelling and computer simulations when those are used to compute numerical solutions. The causal story that, in the case of traditional mechanistic explanations, provides explanatory force by rehearsing how the different entities and activities of the mechanism produce the explanandum phenomenon, appears to break down in the case of more complex and dynamic systems. Indeed, the mathematical techniques that are required to identify or even just approximate solutions in the case of sets of differential equations appear to block any tentative to causally relate the entities and activities of the mechanism to the explanandum phenomenon. ${ }^{10}$

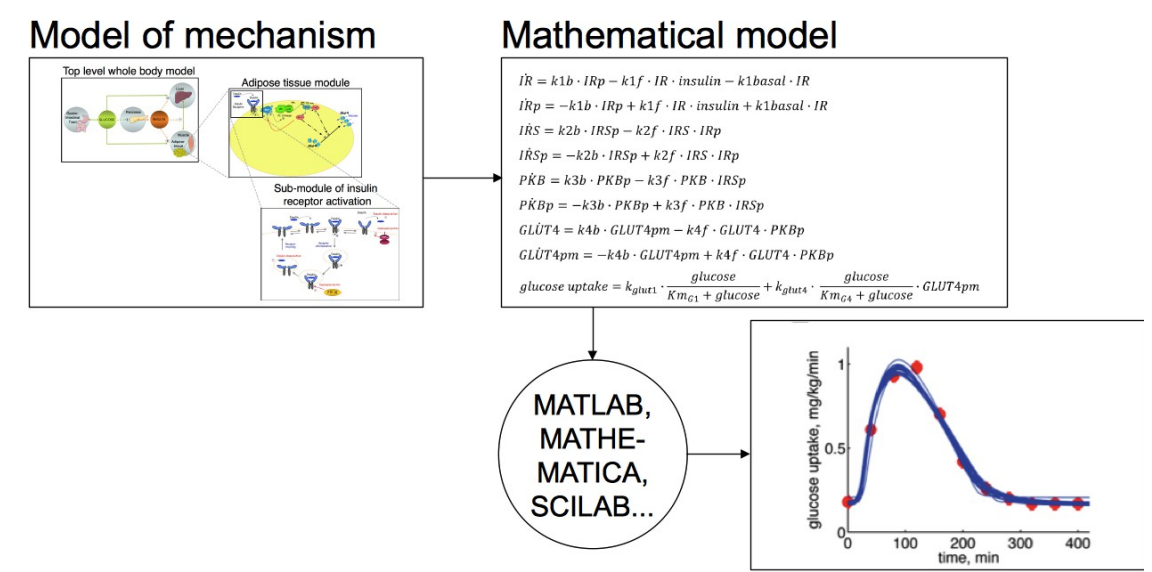

Numerical simulation tool Simulations vs. data

Figure 8. Explanatory steps in the explanation of glucose homeostasis

The causal story condition $(C S)$ therefore is not central in providing

10 Even though the example here only involves ordinary differential equations, we argue that the same argument could be extended to mathematical and numerical methods used in process calculi, boolean networks, Bayesian networks, Petri nets, bipartite graphs, stochastic equations and other ways of formalizing biological models in general. 
explanatory force to DME. Yet the question remains as to where this explanatory force comes from. Our proposal is that the explanatory force comes from the mathematical derivation - analytic or numerical - of the explanandum from the model of mechanism. In other words, not from the satisfaction of the condition $(C S)$ but from the satisfaction of the condition $(M A)$ that consists in providing a mathematical modelling of the model of mechanism such that some or all of its variables and terms correspond to properties of the components and activities of that mechanism, and such that, when analyzed or solved, it leads to a formal reproduction of the explanandum phenomenon. As can be seen in the case of glucose homeostasis, explaining why the concentration of glucose has the specific time-dependent values it has consists (1) in exhibiting a model of mechanism and (2) in asserting that the explanandum phenomenon fits with a mathematical solution of the set of ODE's that describe the mechanism.

The explanatory force of this second component of the explanation does not consist at all in a causal account of how the mechanism produces the phenomenon, but rather in showing that, given a certain set of equations, only specific solution-functions are possible that fit with the explanandum phenomenon. It is the mathematics that tell us how the system variables behave (typically as a function of time), and no causal story. In other words, we grant explanatory power to these solution-functions because they are the result of a mathematical treatment of the model: we are given mathematical warrants that the solution-functions are necessitated by the mathematical modelling. In other words, the facts doing the explaining explain in virtue of being mathematically necessary. Indeed, Lange characterizes the explanatory force of mathematical explanation by the type of modality that mathematical explanations exhibit (Lange, 2012): mathematical explanations typically derive their explanatory force from being modally stronger than laws of nature might actually be, since mathematical explanations are true of any possible world, which is not the case of laws of nature. This is all the more the case for explanations that are solely mathematical in the sense of explaining in virtue on being mathematical in a different way from ordinary explanations that use mathematics ${ }^{11}$. Yet, even if DME are not solely mathematical since they involve not just mathematical models but also models of mechanisms, their explanatory force does come in part from being necessitated by mathematical facts.

\section{Causally-Interpreted Model Explanations (CIME's)}

Explanations of the alleged "mechanistic type" vary significantly in terms of

11 Examples of distinctively mathematical explanations include explanation of why no one ever succeeded in crossing all of the bridges of Königsberg exactly once as specified by Euler, or of why one cannot unknot a trefoil knot, or even of why certain cicada life-periods are prime. See for instance (Baker, 2009, 2014 this volume; Lange, 2012; Pincock, 2007). See also Baetu (2014 this volume), and Breidenmoser and Volkenhauer (2014 this volume). 
the elements that make up their explanatory force, despite sharing the condition of displaying a mechanistic model (condition $M M$ ). Indeed, whereas ME's draw their explanatory force from rehearsing a causal story (condition CS), DME's do not lend themselves to a causal story telling that would be explanatorily relevant, but rather draw their explanatory force from a mathematical model and its resolution or simulation (condition $M A$ ). Therefore DME's are not an extension nor a complement to ME's. Because they draw their explanatory force from a different element, they are rather a different type of explanation altogether.

Another way to see that these two types of explanations are indeed distinct is to notice that they are mobilized in different contexts. Given an explanandum, if the mechanistic model of the explanans is fairly simple, linear (or possibly with very few non-linear elements), then it will be enough for the explanans to complement the mechanistic model by a causal story, and this will do as an explanation. In this case, the explanation will be a mechanistic explanation of the ME type. Yet, if, given another explanandum, the mechanistic model of the explanans becomes complex, with numerous elements and with a high degree of non-linearity, then causal stories do not succeed in bridging the mechanistic model and the explanandum. Rather, they are to be dropped and replaced by a mathematical modelling of the mechanistic model assorted with its resolution/simulation. It is the analytic resolution or the numerical simulation of such mathematical model that enables one to successfully formulate an explanation of the explanandum phenomenon. In other words, the explanatory force of an explanans varies in nature depending on the complexity of the model that it relies on. For simple models, the explanatory force may simply come from displaying a mechanistic model and rehearsing a causal story of how this mechanism works and produces the phenomenon of interest. But for complex models, the explanatory force will come from displaying a mechanistic model, and a corresponding mathematical treatment resulting in a reproduction of the phenomenon of interest. Because the elements that give DME's their explanatory force do not include the elements that make ME's explanatory, DME's are not an extension of ME's.

It is worth noticing that scientists themselves tend to use different terms to refer to these different types of explanations. As has been amply recognized (e.g. Machamer et al., 2000), the concept of mechanism and of mechanistic explanation is often used, in particular in molecular biology. Yet, most of the time - if not always - this is so in the context of fairly simple models. When the models become complex, typically with numerous elements and non-linearity effects, biologists no longer refer to "mechanisms" nor "mechanistic explanations": rather they term their explanations "models". As a matter of fact, this distinction can be seen in the example of insulin research: the explanation of the activation of glucose transporters by insulin is often referred to as a mechanism (Cushman \& Wardzala, 1980; Hoffman \& Elmendorf, 2011; Krook, Wallberg-Henriksson, \& Zierath, 2004); yet, when it comes to explaining the dynamic features of glucose homeostasis, "model" very often replaces 
“mechanism" (e.g. Nyman et al., 2011). ${ }^{12}$

At this point, one may still object that such model explanations are mechanistic in the weaker sense that they nevertheless rely on a model of mechanism as specified by the condition $(M M)$. We could reply that the main argument of our paper was not to show that traditionally construed ME's and DME's did not share any explanatory element at all, but rather to show that they drew their explanatory force from at least one different element, a causal story $(C S)$ in the case of ME, and a mathematical modelling $(M A)$ in the case of DME.

We could also take a further step in the comparison of ME's and DME's. And indeed, we argue that they can be conceived as two extremes of a more encompassing type of explanation that we propose to call "Causally Interpreted Model Explanations" (or CIME's). To support this claim, a further analysis of the different elements of explanatory force that we have singled out above is required. The general idea is that these elements include heterogeneous ideas about formal models on the one hand, and about causal interpretation on the other, and that their reformulation in more homogeneous ways makes it possible to see how ME's and DME's draw their explanatory force differently.

Consider first the $(M M)$ condition of displaying a model of mechanism:

$(M M)$ Displaying a model of mechanism that represents a real mechanism in $\mathrm{S}$, with its entities, activities and spatio-temporal organization

This condition actually includes two distinct elements. First, it includes a condition about displaying a model that has specific characteristics (namely a model with constituents that can be termed entities and activities, and which incorporates a specific spatio-temporal organization). And second, it includes a condition that requires the model (and its constituents and organization) to map onto a real mechanism (and its real constituents and organization). This second condition corresponds to an interpretation of the model; and because the entities and activities of mechanisms are individuated on the basis of their causal roles, it is a causal interpretation of the model.

Consider now the condition $(M A)$ of displaying a mathematical model:

$(M A)$ Displaying a mathematical model such that some or all of its variables and terms correspond to properties of identified parts and operations of the mechanism, and providing mathematical warrants that its resolution/simulation fits $\mathrm{P}$.

This condition of explanatory force also mixes requirements that concern a model on the one hand, and its interpretation on the other. The condition indeed requires that a model be presented, and this model be of a mathematical type. The condition also requires that some of the variables and terms of the mathematical model correspond to parts and operations - or entities and activities - of the underlying real mechanism that is supposed to produce the explanandum phenomenon. This second condition is again a condition of

12 Because the explanatory force depends on the complexity of the model, and because complexity is relative to cognitive faculties, we acknowledge here the fact that the nature of the explanatory force will vary from cognitive context to cognitive context. 
interpretation of the model, and this interpretation is typically be required to be causal. Lastly, the condition requires that one should be given warrants that the mathematical solutions of the model follow, either analytically through deductions or numerically through simulations, from the model, and that these solutions fit the explanandum phenomenon. Let's call this last condition a condition of derivation of the explanandum phenomenon.

Lastly, consider the causal story condition $(C S)$ that plays such a key role in ME's when conjoined with the model of mechanism condition $(M M)$ :

(CS) Rehearsing a causal story that enumerates the cause-effect relationships taking place in the mechanism up to the production of the phenomenon $\mathrm{P}$.

The requirements of $(C S)$ can be articulated along two components. First $(C S)$ requires the identification of causes and effects up to the explanandum phenomenon P. It therefore includes a causal interpretation of what is captured by the model of mechanism and which is supposed to happen in the real mechanism. Second, $(C S)$ requires a "story" that links the different causes to their effects, and ultimately the mechanism to the explanandum phenomenon. This story typically includes the verbs that correspond to the activities of the entities of the mechanism. However, should a functional (mathematical) formulation be made available, such story could easily be transcribed into simple mathematical (or even boolean) expressions. ${ }^{13}$ It is therefore entirely possible to rephrase the causal story as a succession of simple mathematical derivations, up to a mathematical expression of the explanandum phenomenon. For this reason, part of the $(C S)$ requirements indeed consist of some form of derivation requirement.

To sum up, the above unpacking of (MM), (MA) and (CS) shows that these requirements of explanatory force draw somehow differently on three main elements: a model, a causal interpretation and a derivation. We propose to define the notion of "Causally Interpreted Model Explanations" (CIME's) around these three elements as follows:

A causally interpreted model explanation (CIME) explains a phenomenon (P) produced by a system $(\mathrm{S})$ in virtue of the following three necessary and sufficient conditions:

$(M)$ Displaying a model of $\mathrm{S}$ that includes variables and functions defined over these variables,

(CI) Providing a causal interpretation of these variables and functions (for instance by means of a manipulationist account of causation),

(D) Deriving, through a mathematical treatment (be it analytical or numerical), the explanandum phenomenon $\mathrm{P}$ from the model.

So construed, CIME's explain a phenomenon neither in virtue of displaying

13 To see how this could be done, remember that a manipulationist account of causation is often assumed to be compatible with the mechanistic account of explanation. Such an account of causation is typically articulated around (causal) variables and makes it possible to transcribe the (cause-effect) relationships between these variables into mathematical functions. See for instance (Woodward, 2003). 
a mechanism nor in virtue of providing a causal account, but in virtue of mathematically showing how the explanandum can be analytically or numerically derived from a model whose variables and functions can be causally interpreted. ${ }^{14}$

ME's and DME's can then be seen as two different limiting cases of CIME's, even if no sharp delineation may actually exist between the two. Indeed, a ME is a limit case of CIME in which (1) the model specified by $(M)$ is relatively simple and the causal interpretation provided in $(C I)$ makes it possible to construe the model as a mechanism, hence fulfilling the $(M M)$ condition, and (2) the derivation of the explanandum from the model is also simple enough typically involving linear relations - so as to be reformulated into a causal story - typically with verbs transcribing functional relations into activities, and other terms transcribing variables and their values into entities - thereby fulfilling $(C S)$. Consider now a DME. We argue that a DME can be construed as a limit case of CIME in which (1) the model specified by $(M)$ is relatively complex, involving in particular non-linear functions, (2) the causal interpretation provided by $(C I)$ is likely to be less exhaustive compared to a ME, possibly involving non-interpreted parameters, and (3) the derivation of the explanandum from the model is done through relatively intricate analytical or numerical methods and, as a result, does not lend itself to a reformulation as a causal-story (see Fig. 8).

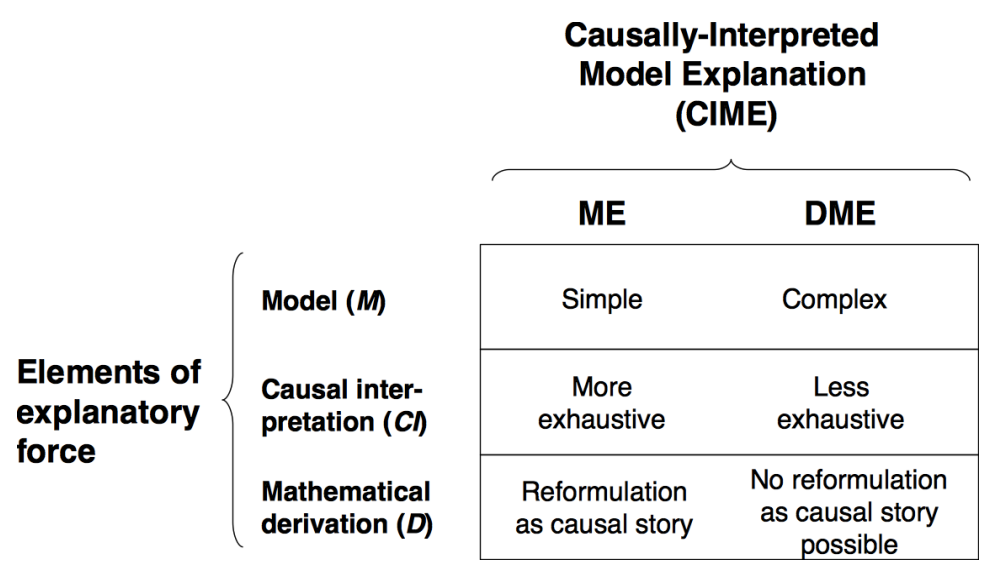

Figure 8. Elements of explanatory force of Causally-Interpreted Model Explanations (CIME's), in relationship to Mechanistic Explanations (ME's) and Dynamic Mechanistic Explanations (DME's).

14 This account is characterized in terms of models that rely on "variables" and "functions". We believe - though we do not show it here - that such characterization could be extended to cover other more specific ways of construing models, be they for instance in terms of set-theoretic predicates or phase-state. Note also that we do not mean that all variables in the model be causally interpreted. It is indeed possible that some of the variables do not receive any causal interpretation. This may also be the case for specific parameters and coefficients used in the model. 
An important point is the fact that supplying a causal-story is no longer seen central in providing explanatory force: the key element of explanatory force is the mathematical derivation. While this derivation can be reformulated in causal language in some of the simpler cases, we have to acknowledge that in the more complex cases, such derivation involves many intricate mathematical treatments - including numerous theorems and proofs, approximating techniques, numerical simulation tools etc. - that make any causal retranscription hopeless. Yet, the derivation does convey the type of explanatory force that is expected from a proper explanation. And it does so in virtue of the mathematical warrants that it provides and that make the explanandum follow from the model with mathematical necessity.

We also argue that providing a mechanism per se is also not so central when it comes to explanatory force. What matters more generally is the presence of a model and of its causal interpretation. And while we recognize the fact that, in many relatively simple cases, such model and causal interpretation can be transcribed into a model of mechanism, we also want to underline the fact that, in numerous complex cases, the explanatory does not come from a model of mechanism but from a model - more generally construed - and the causal interpretation that accompanies it. The causal interpretation in particular is seen as providing the crucial link between the model and biological reality. But it is the analytical/numerical derivation that links the model to the explanandum, not a causal story.

\section{Conclusion}

In this contribution, we aimed at getting at the source of the explanatory force that is claimed to be that of mechanistic explanations. When looking at simple cases of mechanisms in biology, such as the insulin-induced relocalization of glucose transport systems, it appears that this explanatory force can be understood as coming from a model of mechanism and a causal story that tells how the mechanism produces the explanandum phenomenon. However, when biological systems tend to become complex, as in the case of glucose homeostasis, mathematical modelling becomes a necessary explanatory component that turns mechanistic explanations into dynamic mechanistic explanations. It is important to note that the explanatory force of such explanations does not any longer come from the rehearsing of a causal story, but from mathematical warrants that the explanandum follows from the model. To account for this disparity of explanatory force, we have proposed a more general pattern of "Causally Interpreted Model Explanations" according to which explanations draw their explanatory force from a model, a causal interpretation that links the model to biological reality, and a mathematical derivation that links the model to the explanandum phenomenon. Mechanistic explanations and dynamic mechanism explanations can then be construed as different limit cases of causally interpreted model explanations. Nevertheless, 
providing causal stories is not as central to explanatory force as is mathematical derivation.

\section{References}

Baldan, P., Cocco, N., Marin, A., \& Simeoni, M. (2010). Petri nets for modelling metabolic pathways: a survey. Natural Computing (Vol. 9, pp. 955-989). doi:10.1007/s11047-010-9180-6

Bechtel, W. (2011). Mechanism and Biological Explanation*. Philosophy of Science, 78(4), 533-557. doi:10.1086/661513

Bechtel, W., \& Abrahamsen, A. (2010). Dynamic mechanistic explanation: computational modeling of circadian rhythms as an exemplar for cognitive science. Studies in History and Philosophy of Science Part A, 41(3), 321333. doi:10.1016/j.shpsa.2010.07.003

Bechtel, W., \& Abrahamsen, A. a. (2012). Thinking Dynamically About Biological Mechanisms: Networks of Coupled Oscillators. Foundations of Science. doi:10.1007/s10699-012-9301-z

Belsham, G. J., Brownsey, R. W., Hughes, W. A., \& Denton, R. M. (1980). Anti-insulin receptor antibodies mimic the effects of insulin on the activities of pyruvate dehydrogenase and acetylCoA carboxylase and on specific protein phosphorylation in rat epididymal fat cells. Diabetologia, 18(4), 307-312. doi:10.1007/BF00251011

Brännmark, C., Palmér, R., Glad, S. T., Cedersund, G., \& Strålfors, P. (2010). Mass and information feedbacks through receptor endocytosis govern insulin signaling as revealed using a parameter-free modeling framework. The Journal of biological chemistry, 285(26), 20171-9. doi:10.1074/jbc.M110.106849

Capotosti, F., Guernier, S., Lammers, F., Waridel, P., Cai, Y., Jin, J., ... Herr, W. (2011). O-GlcNAc Transferase Catalyzes Site-Specific Proteolysis of HCF-1. Cell, 144(3), 376-388.

Carracedo, A., \& Pandolfi, P. (2008). The PTEN-PI3K pathway: of feedbacks and cross-talks. Oncogene, 27(41), 5527-5541.

Combettes-Souverain, M., \& Issad, T. (1998). Molecular basis of insulin action. Diabetes \& metabolism, 24(6), 477.

Craver, C. F. (2007). Explaining the brain: Mechanisms and the mosaic unity of neuroscience. Book. Oxford University Press.

Cushman, S. W., \& Wardzala, L. J. (1980). Potential mechanism of insulin action on glucose transport in the isolated rat adipose cell. Apparent translocation of intracellular transport systems to the plasma membrane. Journal of Biological Chemistry, 255(10), 4758-4762. 
Dalla Man, C., Rizza, R. a, \& Cobelli, C. (2006). Mixed meal simulation model of glucose-insulin system. Conference proceedings : ... Annual International Conference of the IEEE Engineering in Medicine and Biology Society. IEEE Engineering in Medicine and Biology Society. Conference, 1(10), 307-10. doi:10.1109/IEMBS.2006.260810

De Jong, H. (2002). Modeling and simulation of genetic regulatory systems: a literature review. Journal of computational biology : a journal of computational molecular cell biology, 9(1), 67-103. doi:10.1089/10665270252833208

Deville, Y., Gilbert, D., van Helden, J., \& Wodak, S. (2003). An overview of data models for the analysis of biochemical pathways. Brief Bioinform, $4(3), 246-259$.

Gerhardt, C. C., Gros, J., Strosberg, A. D., \& Issad, T. (1999). Stimulation of the extracellular signal-regulated kinase $1 / 2$ pathway by human beta- 3 adrenergic receptor: new pharmacological profile and mechanism of activation. Molecular pharmacology, 55(2), 255-262.

Glennan, S. S. (2002). Rethinking mechanistic explanation. Philosophy of Science, 69(3), S342-S353.

Glennan, S. S. (2005). Modeling mechanisms. Studies in History and Philosophy of Science Part C: Studies in History and Philosophy of Biological and Biomedical Sciences, 36(2), 443-464. doi:10.1016/j.shpsc.2005.03.011

Hoffman, N. J., \& Elmendorf, J. S. (2011). Signaling, cytoskeletal and membrane mechanisms regulating GLUT4 exocytosis. Trends in Endocrinology \& Metabolism, 22(3), 110-116.

Hubbard, S. R. (1997). Crystal structure of the activated insulin receptor tyrosine kinase in complex with peptide substrate and ATP analog. The EMBO journal, 16(18), 5572-5581.

Hubbard, S. R., Wei, L., Ellis, L., \& Hendrickson, W. A. (1994). Crystal structure of the tyrosine kinase domain of the human insulin receptor. Nature, 372(6508), 746.

Hunter, T. (1995). When is a lipid kinase not a lipid kinase? When it is a protein kinase. Cell, 83(1), 1-4.

Issad, T., Masson, E., \& Pagesy, P. (2010). O-GlcNAc modification, insulin signaling and diabetic complications. Diabetes \& metabolism, 36(6), 423435.

Issad, Tarik, Combettes, M., \& Ferre, P. (1995). Isoproterenol Inhibits InsulinStimulated Tyrosine Phosphorylation of the Insulin Receptor Without Increasing its Serine/Threonine Phosphorylation. European Journal of Biochemistry, 234(1), 108-115. 
Issad, Tarik, \& Kuo, M. (2008). O-GlcNAc modification of transcription factors, glucose sensing and glucotoxicity. Trends in Endocrinology \& Metabolism, 19(10), 380-389.

Kaplan, D. M., \& Bechtel, W. (2011). Dynamical Models: An Alternative or Complement to Mechanistic Explanations? Topics in Cognitive Science, 3(2), 438-444. doi:10.1111/j.1756-8765.2011.01147.x

Kasuga, M., Zick, Y., Blith, D. L., Karlsson, F. A., Häring, H. U., \& Kahn, C. R. (1982). Insulin stimulation of phosphorylation of the beta subunit of the insulin receptor. Formation of both phosphoserine and phosphotyrosine. Journal of Biological Chemistry, 257(17), 9891-9894.

Kasuga, Masato, Zick, Y., Blithe, D., Crettaz, M., \& Kahn, C. (1982). Insulin stimulates tyrosine phosphorylation of the insulin receptor in a cell-free system. Nature, 298, 667-669.

Kiselyov, V. V, Versteyhe, S., Gauguin, L., \& De Meyts, P. (2009). Harmonic oscillator model of the insulin and IGF1 receptors' allosteric binding and activation. Molecular systems biology, 5(243), 243. doi:10.1038/msb.2008.78

Krook, A., Wallberg-Henriksson, H., \& Zierath, J. R. . (2004). Sending the signal: molecular mechanisms regulating glucose uptake. Med Sci Sports Exerc, 36(7), 1212-1217.

Lacasa, D., Boute, N., \& Issad, T. (2005). Interaction of the insulin receptor with the receptor-like protein tyrosine phosphatases PTPalpha and PTPepsilon in living cells. Mol. Pharmacol., (4), 1206-1213.

Lange, M. (2012). What Makes a Scientific Explanation Distinctively Mathematical? The British Journal for the Philosophy of Science, 0, 1-27. doi:10.1093/bjps/axs012

Machamer, P., Darden, L., \& Craver, C. (2000). Thinking about mechanisms. Philosophy of science.

Mandel, J., Palfreyman, N., Lopez, J., \& Dubitzky, W. (2004). Representing bioinformatics causality. Brief Bioinform, 5(3), 270-283.

Moule, S. K., Welsh, G. I., Edgell, N. J., Foulstone, E. J., Proud, C. G., \& Denton, R. M. (1997). Regulation of protein kinase B and glycogen synthase kinase- 3 by insulin and $\beta$-adrenergic agonists in rat epididymal fat cells Activation of protein kinase B by wortmannin-sensitive andinsensitive mechanisms. Journal of Biological Chemistry, 272(12), 77137719 .

Nouaille, S., Blanquart, C., Zilberfarb, V., Boute, N., Perdereau, D., Roix, J., ... Issad, T. (2006). Interaction with Grb14 results in site-specific regulation of tyrosine phosphorylation of the insulin receptor. EMBO Reports, (7), $512-518$. 
Nyman, E., Brännmark, C., Palmér, R., Brugård, J., Nyström, F. H., Strålfors, P., \& Cedersund, G. (2011). A hierarchical whole-body modeling approach elucidates the link between in Vitro insulin signaling and in Vivo glucose homeostasis. The Journal of biological chemistry, 286(29), 26028-41. doi:10.1074/jbc.M110.188987

Smith, C., Rubin, C., \& Rosen, O. (1980). Insulin-treated 3T3-L1 adipocytes and cell-free extracts derived from them incorporate 32P into ribosomal protein S6. Proceedings of the ....

Vazquez, F., \& Sellers, W. R. (2000). The PTEN tumor suppressor protein: an antagonist of phosphoinositide 3-kinase signaling. Biochimica et biophysica acta, 1470(1), M21.

Virkamäki, A., Ueki, K., \& Kahn, C. R. (1999). Protein-protein interaction in insulin signaling and the molecular mechanisms of insulin resistance. $J$. Clin. Invest, 103(7), 931-943.

Wiekert, W. (2002). Modelling and simulation: tools for metabolic engineering. J Biotechnol, 94(1), 37-63.

Woodward, J. (2003). Making Things Happen: A Theory of Causal Explanation: A Theory of Causal Explanation. Oxford University Press, USA. 